\section{Euthanasie: le point de vue de l'éthique médicale judaïque}

\section{Y. Nordmann}

Traduction: Dr M. Wenner, Villeneuve VD

Deutsch erschienen in Nr. 46/2001

\section{Préambule}

On discute depuis longtemps de la légalisation de l'euthanasie active; ce débat a pris récemment une tournure nouvelle, non dénuée de dangers. En Hollande, les derniers obstacles moraux sont récemment tombés avec la légalisation de l'euthanasie active. En Suisse, la discussion sur le même sujet, appelé aussi "assistance médicale au suicide", provoque de telles vagues, qu'il faut craindre une évolution analogue. Au moment où l'on apprenait l'acte d'un jeune infirmier, qui a tué "par compassion" de nombreuses personnes dans différentes institutions, une commission du Conseil des Etats, emmenée par Franco Cavalli (conseiller national et oncologue) a fait la proposition de légaliser également l'euthanasie en Suisse. Où cela va-t-il nous conduire? Le texte qui suit veut apporter à l'indispensable discussion quelques réflexions provenant de l'éthique médicale judaïque. En outre, il espère introduire le lecteur dans le mode de pensée de cette éthique, qui jusqu'à présent, n'est que peu connue.

\section{Introduction à l'éthique médicale judaïque}

Dans le quotidien médical, les valeurs religieuses se retrouvent fréquemment au premier plan des préoccupations éthiques et des processus de décision, surtout lorsqu'il s'agit de questions touchant aux limites de la vie. Dans le monde juif, le système éthique tente de résoudre les problèmes complexes qui se posent, en se basant sur la tradition millénaire et sur sa reconnaissance dans de très larges cercles. Ceci est probablement dû au fait que dans la religion juive, il n'y a pas de frontière marquée entre l'éthique et la loi: la loi c'est l'éthique, et inversement. Pour connaître

Correspondance:

Dr Yves Nordmann

Mutschellenstrasse 55

CH-8038 Zurich l'éthique médicale juive, il faut donc connaître la loi juive. La religion juive se base sur deux fondements: la loi écrite et la transmission orale. La loi écrite, appelée habituellement la Bible, se compose de trois parties: la Tora, les Neviim et les Ketuvim, mais la Tora est la plus importante: c'est la base de la foi juive qui a recueilli dans cinq livres l'enseignement divin que Moïse reçut au Mont Sinaï. La loi juive ne se compose pas que d'une partie écrite: elle est complétée par la tradition orale, sans laquelle beaucoup d'éléments écrits ne seraient pas compréhensibles. Sur le Mont Sinaï, Dieu a remis à Moïse et au peuple juif cette tradition orale en même temps que la loi. La tradition orale est restée intacte jusqu'au temps présent. $\mathrm{Au}$ deuxième siècle apr. J.-C., on commença à transcrire la tradition orale dans un ouvrage appelé Talmud, qui fut connu bien au delà de la religion juive. Dorénavant, le Talmud joue un rôle extrêmement important dans la tradition juive, telle une «encyclopédie» de l'ensemble des connaissances juives.

\section{Qu'entend-on par Halacha?}

Tout problème traité dans le cadre de la loi juive (la Halacha) ne peut se faire sans l'étude des sources, la Tora, le Talmud et leurs commentaires. Il n'est pas possible de résoudre les problèmes moraux en médecine sur la base de sentiments ou de valeurs personnels, mais une analyse "halachique» est exigée, effectuée par une autorité rabbinique dont la décision devra être respectée par la suite. Certes, il ne suffit pas, pour le rabbin, d'une connaissance de base des sources "halachiques»; toutes les circonstances faisant partie du cas spécifique étudié doivent être considérées. Un point central doit être précisé ici: la religion juive n'est pas dirigée par une autorité suprême (comme par exemple dans le catholicisme), mais elle comporte différents "Poskim», soit des "porteurs de décisions»: des rabbins extrêmement savants et très qualifiés, qui ont acquis une notoriété mondiale au travers de leurs décisions et prises de position. Le "porteur de décisions" n'est pas nommé à cette fonction, mais il «reçoit» plutôt ce rôle par son autorité personnelle et professionnelle, manifestée essentiellement par le fait qu'il reçoit des demandes de tous les points du globe. La plupart des rabbins et la plus grande partie de la population juive orthodoxe mondiale accepte les décisions d'une autorité rabbinique de ce type.

Aujourd'hui, il s'agit entre autres de Rav Joseph Elijaschiv et Rav Elieser Jehuda Waldenberg (spécialement pour les questions médicales); tous deux vivent en Israël, ont obtenu le rôle de "Poskim» et exercent en tant que tels. Il peut arriver que différents "Poskim» prennent des décisions différentes pour les mêmes questions, malgré le fait qu'ils disposent d'une source commune pour analyser les problèmes (la Tora). Comment cela peut-il être expliqué? Les mots de la Tora eux-mêmes sont inamovibles; leur interprétation cependant, dépend de l'intelligence humaine, comme il est dit dans la Bible (Deutéro- 
nome, 30: 12): "lo baschamaim hi», c'est à dire "la Tora ne se trouve pas au Ciel», mais dans les mains de l'Homme qui l'interprétera et la mettra en pratique. Il est donc possible, dans l'enseignement juif, que deux conclusions opposées, émises par diverses autorités sur la base de la même source soient exprimées. Laquelle sera la bonne? Les deux! Car selon le Talmud, il est dit (Gittin, 6b): "Ceci et cela sont les mots du Dieu vivant». Il faut cependant préciser que la foi juive contient plusieurs principes de base incontestés, mentionnés explicitement dans la Tora. En lien avec la problématique de l'euthanasie, quelquesuns de ces principes vont être présentés.

\section{Aspects fondamentaux sur l'euthanasie (du point de vue judaïque)}

L'interdiction de tuer

La Tora prescrit de façon générale l'interdiction de tuer un être humain. Une seule situation d'exception est celle de la légitime défense: il est autorisé, afin d'éviter sa propre mort ou celle d'une personne innocente, de tuer un meurtrier potentiel (et seulement lorsqu'il n'y a pas d'autre solution!). Donc, la mise à mort d'une personne qui ne provoque aucune menace pour les autres, est considéré comme un meurtre, qu'il s'agisse d'un nouveau-né, d'une personne adulte saine ou d'un mourant [1]. L'interdiction de tuer est également valable pour le suicide, même si cet acte a été commis dans l'intention de sauver la vie d'une autre personne.

\section{La vie vue comme un don}

L'Homme n'a aucun droit absolu sur son propre corps, puisque Dieu a mis à sa disposition, pour un certain temps, un corps et une âme; chaque personne est donc responsable de prendre soin de ce don [2]. Du point de vue judaïque, il n'est donc pas autorisé de disposer librement de son corps, de se blesser volontairement ou de se suicider.

La valeur infinie de la vie humaine

Dans la foi juive, un principe fondamental est constitué par le concept que la vie humaine est sacrée, intouchable, et possède une valeur infinie. Le rabbin Lord Immanuel Jokobovits (1921-1999), ancien grand-rabbin de Grande-Bretagne et expert dans le domaine de l'éthique médicale judaïque, a fait une description très pertinente de ce principe:

"[...] la valeur de la vie humaine est infinie et audelà de toute mesure, à tel point que toute durée de vie - même s'il ne s'agit que d'une heure ou d'une seconde - a très précisément la même valeur que septante ans; de même, n'importe quelle fraction de l'infinité sera indivisible, et restera infinie. Ainsi, mettre fin à la vie d'un patient très affaibli constitue exactement le même crime que de tuer une personne jeune, en bonne santé et qui aurait encore des dizaines d'années de vie devant elle.» [3]
Ainsi, la position de base de l'éthique médicale judaïque face à l'euthanasie est très clairement définie: la valeur infinie de la vie humaine constitue un acquis indispensable à une société dotée d'une haute valeur morale. De même que la plus petite partie de l'infinité est également une infinité, chaque instant de la vie humaine a la même valeur infinie. C'est ainsi qu'une personne qui n'a plus que quelques jours ou quelques heures à vivre n'a pas moins de valeur qu'une personne qui va vivre encore de nombreuses années, car la valeur infinie de la vie humaine ne peut pas perdre son caractère absolu, donc ne peut être relativisée. On ne peut pas évaluer l'utilité d'une personne par rapport à la société en fonction de son espérance de vie ou de son état de santé. Des critères extrêmement subjectifs pourraient être choisis afin de déterminer la valeur ou la non-valeur d'une vie humaine. Cela nous conduirait à une catégorisation des personnes en deux catégories: ceux qui ont le droit de vivre et ceux qui n'ont plus le droit de vivre, d'où le danger d'une échelle de valeurs qui a existé dans un des chapitres les plus sombres de l'histoire de l'Humanité. Il n'est donc pas étonnant que ce soit la religion juive qui ait une attitude aussi "sensible» par rapport à la question de l'euthanasie. Le philosophe Jeshajahu Leibovitz exprime cela en des termes impressionnants [4]: "La question de savoir si il y a une vie digne d'être vécue ne doit pas exister".

\section{Définition et statut du mourant du point de vue judaïque}

Comment se définit la personne en fin de vie d'après les sources classiques halachiques? Dans le Talmud et parmi les autorités rabbiniques, le mourant est nommé goses, ce qui désigne une personne qui est toute proche de la rencontre avec la mort. Une définition plus exacte est difficile, même si différents savants ont essayé de la préciser: Rabbi Mosche Isserles (Rema, $16^{\mathrm{e}} \mathrm{s}$.) par exemple dit qu'un goses est un «patient qui est si proche de la mort qu'il régurgite du mucus, parce qu'il a d'énormes difficultés à respirer" (Commentaire sur Even Haeser, 121:7, et Choschen Mischpat, 211:2; traduction personnelle). Pour Rav Bleich, un rabbin contemporain, la "définition clinique» est insuffisante pour notre temps. Pour lui, un goses est une personne qui n'a en aucun cas plus de septante-deux heures de vie devant lui, même avec l'aide de médicaments [5].

En dépit des incertitudes au sujet de la définition de la personne mourante, les anciennes sources ont précisé clairement le statut du mourant. Dans le paragraphe Semachot, 1:1-4, du Talmud il est écrit: "Celui qui est en fin de vie sera considéré en tous points comme une personne vivante. On ne doit donc ni lui mettre un bandage sous la mâchoire, ni colmater ses orifices [...]. On ne doit pas le mobiliser [...]. On ne doit pas fermer les yeux d'une personne mourante. Celui qui la touche et la déplace, celui-là fait couler le sang»: comme aimait le dire Rabbi Meir: 
"on peut comparer ce temps à une flamme très faible: dès qu'une personne la touche, elle s'éteint». Et aussi: "celui qui ferme les yeux d'une personne mourante sera considérée comme s'il lui avait enlevé son âme [...]». Ces textes sont très impressionnants, ils montrent quels sont les préceptes en rapport avec la fin de la vie: il est interdit d'accélérer la mort, en raison du principe absolu de la sainteté de la vie humaine. Donc, une personne en fin de vie ne peut pas être touchée ni déplacée (font exception à cette règle les actes médicaux ou les soins indispensables pour le patient).

Même si les lois sont très sévères, il y a cependant quelques écrits juifs qui peuvent justifier l'euthanasie passive. Il faut insister sur le fait que l'on parle toujours d'un goses, donc d'une personne qui est très proche de la mort. Une première source se trouve dans le «livre des fidèles» de Rabbi Jehuda ben Samuel, qui écrivait environ dans les années 1200 apr. J.-C.: «[...] lorsqu'une personne est en fin de vie, alors que dans le voisinage quelqu'un fend du bois en faisant un bruit tel que l'âme du mourant ne peut pas s'envoler, il faut que l'on chasse ce bûcheron". [6] Sur ce passage, Rabbi Mosche Isserles (16 ${ }^{\mathrm{e}} \mathrm{s}$.) a fait ce commentaire: "[...] lorsqu'il y a quoi que ce soit qui empêche l'âme de s'échapper, comme par exemple un bruit violent à proximité de la maison du patient [...], et que ce bruit empêche l'âme de s'échapper, alors il est permis d'éloigner cet obstacle, car de cette manière, il n'y a pas de gestes effectués (sur le patient), mais il y a simplement un obstacle qui est enlevé» [7]. Du point de vue judaïque, il est donc permis, pour un patient très proche de la mort (un goses), et qui ne peut pas mourir, d'éloigner l'élément qui l'empêche de décéder, et ainsi de laisser arriver la mort. Une autre source, probablement plus éloignée du thème de l'euthanasie, se trouve dans le paragraphe Ketubot (104a) du Talmud, où il est signalé que Rabbi Jehuda Hanassi ( $2^{\mathrm{e}} \mathrm{s}$.), atteint lui-même d'une maladie incurable et très débilitante, était soigné par une servante, une femme décrite dans la littérature rabbinique comme une personne de grande foi et de haute moralité. Cette servante aurait prié pour la mort de Rabbi Jehuda Hanassi, afin que celui-ci soit enfin délivré de ses souffrances. Sur la base de cette description, Rabbenu Nissim de Gerondi ( $13^{\mathrm{e}}$ s., commentaire au sujet de Nedarim 40a) ajoute: "A mon avis [...], il y a des circonstances dans lesquelles on devrait prier pour la mort d'un malade, par exemple, lorsque la personne souffre énormément, et que sa survie ne puisse plus être espérée, comme c'était le cas pour Rabbi Jehuda Hanassi et pour sa servante». Le Rabbin Lord Jakobovits décrivait ce commentaire de Rabbenu Nissim comme une grande concession en faveur de l'«euthanasie», car dans la foi juive, les prières jouent un rôle primordial, et nul ne doute de leur pouvoir.

\section{Attitude actuelle des autorités rabbiniques}

La discussion actuelle autour de l'attitude judaïque par rapport à l'euthanasie a été résumée par le
Rabbin Lord Jakobovits dans son ouvrage «Jewish Medical Ethics» de la façon suivante: "Toute forme d'euthanasie active est formellement interdite par la loi juive. Elle est équivalente à un meurtre [...], mais en même temps, la Halacha autorise ou pourrait même exiger d'éloigner un facteur extérieur au patient ou sur le patient lui-même (pour autant que le patient ne soit pas déplacé), facteur qui pourrait prolonger l'agonie" [8]. Lord Jakobovits insiste sur le fait que cette règle n'a de validité que pour un goses: ainsi, elle ne s'applique pas pour l'euthanasie passive, chez un patient qui a encore des semaines ou des mois de vie devant lui.

Rav Elieser Jehuda Waldenberg, qui fait autorité parmi les rabbins pour les questions médicales, traite dans son ouvrage médical halachique "Ziz Elieser" de la problématique de l'euthanasie de manière approfondie. Rav Waldenberg défend l'opinion que toute personne qui se conforme aux lois juives doit entreprendre tout ce qui est en son pouvoir afin de maintenir en vie une personne mourante, même si cela ne devait être possible pour un temps court. Ceci devrait également se faire pour tout patient, même s'il souffre beaucoup ou qu'il demande à ce qu'on abrège ses jours [9]. Il est vrai que la loi juive autorise de donner à des patients en fin de vie des opiacés comme de la morphine, même si la médication devait accélérer la mort, cependant jamais avec l'intention de le faire, donc uniquement à titre antalgique [10]. Rav Waldenberg recommande par ailleurs d'administrer des transfusions sanguines, de l'oxygène, des antibiotiques, ainsi que des nutriments par voie orale ou parentérale également à un patient incurable et ceci jusqu'à sa mort [11]. Rav Schlomo Salmen Auerbach, une des plus grandes autorités rabbiniques du $20^{\mathrm{e}}$ siècle, souligne également qu'il est impératif d'administrer à un malade en fin de vie de la nourriture et de l'oxygène, ceci même si c'est contre la volonté du patient [12].

Rav Bleich a décrit ainsi l'attitude judaïque par rapport à l'euthanasie: "La pratique de l'euthanasie, quelle soit active ou passive, est en contradiction avec la doctrine judaïque. Toute action ayant pour but d'accélérer la mort d'un patient est un meurtre d'après la loi juive, même si la mort ne survient que quelques instants plutôt que naturellement. Même si les motifs de la personne étaient honorables, qu'elle a fait preuve de charité, son action était un meurtre [...]. Seul le Créateur de ce monde qui nous a donné la vie comme un cadeau a le droit de reprendre cette vie même lorsque cette vie est devenue plus une charge qu'une bénédiction». [5] Rav Bleich insiste sur la situation particulière d'un goses: la mort ne doit pas être accélérée, mais il est possible, dans certaines circonstances, de renoncer à instaurer un traitement. Rav Bleich cite également quelques auteurs qui acceptent non seulement l'abstention thérapeutique, mais qui pensent que toute action qui prolongerait la vie d'un goses est interdite. Par exemple, Rav Mosche Feinstein commente: "Il est certainement interdit de tenter de prolonger la vie d'une personne mourante lorsque cela aboutirait à des douleurs et des souf- 
frances supplémentaires. Il est également interdit de raccourcir la vie, et celui qui commet cet acte est un meurtrier, même s'il agit à la demande et sur l'insistance du patient [...]». [13] Le Professeur Abraham, expert contemporain pour les questions médicales et halachiques, a tenté de rassembler des recommandations en rapport avec la problématique de l'euthanasie du point de vue judaïque [14]; celles-ci sont résumées comme suit:

1. En principe, tous les patients, indépendamment de leur état, doivent être traités avec des nutriments, des liquides, de l'oxygène et d'autres éléments qui maintiennent la vie, ceci même si ces éléments doivent être administrés par des voies inhabituelles.

2. Les patients atteints de maladies chroniques gravissimes et qui ne sont pas en fin de vie doivent être traités exactement comme tous les autres patients.

3. Les patients avec des maladies terminales qui sont en fin de vie (goses), doivent également être traités comme tous les autres patients. Au cas où un goses subit un arrêt cardiaque ou respiratoire, ou d'autres complications qui pourraient nécessiter des soins plus intensifs, et qui pourraient le péjorer encore plus, il faut:

a) Lorsque l'arrêt cardiaque ou respiratoire est dû à la maladie terminale, c'est à dire se produit dans le cadre d'une évolution inéluctable, il n'est pas indispensable de tenter une réanimation, cela pourrait même être une erreur.

b) Lorsque l'arrêt cardiaque ou respiratoire (ou d'autres complications) surviennent de façon impromptue et indépendamment de la maladie terminale, un traitement complet doit être instauré, même chez un goses, comme cela se ferait pour n'importe quel patient. Cette règle n'est cependant valable qu'à condition de ne pas accentuer les souffrances du patient et sa lutte contre la mort.

\section{Conséquences pour la discussion actuelle}

Dans le débat sur la légalisation de l'euthanasie active (ou suicide assisté médicalement), il s'avère que les promoteurs d'une telle réglementation sont en contradiction avec les principes centraux et fondamentaux de l'éthique médicale judaïque. Ils méprisent l'interdiction de tuer et le principe biblique du don de la vie. Ce dernier concept semble en contradiction avec la tendance actuelle de l'éthique sécularisée, où l'autonomie du patient a acquis une valeur toujours plus grande. Il s'agit d'une grave erreur de considérer l'intention de suicide d'un individu comme une affaire privée, ne concernant pas la société dans son ensemble. Dans ce contexte, différentes études au sujet du suicide assisté ont montré que c'étaient sur- tout les personnes âgées et modestes (souvent déjà marginalisées) qui auraient été encouragées à faire usage de leur "droit de mourir». Il est totalement inadmissible d'exercer une pression morale sur ceux qui désirent continuer de vivre, malgré leurs souffrances et les coûts engendrés par leur traitement. La question se pose également de savoir à qui servirait la légalisation de l'euthanasie active, respectivement du suicide assisté médicalement: à l'individu qui souffre, qui a besoin de notre soutien émotionnel? Ou à la société elle-même, qui cherche à résoudre les problèmes des coûts de la santé et de la limitation des ressources? Pourrait-on ainsi se débarrasser de patients "gênants" d'une façon "simple»? Le mépris de l'interdiction de tuer est critiquée par ailleurs par d'autres autorités: par exemple, le président de la Chambre Médicale allemande (Prof. Jörg-Dietrich Hoppe), au sujet de l'évolution en Hollande: "La décision du Parlement néerlandais de supprimer l'interdiction de tuer dans certains cas et d'accepter l'euthanasie assistée médicalement se heurte aux fondements d'une société humaine".

Les expériences faites jusqu'à présent nous montrent que la légalisation de l'euthanasie active a provoqué bien des problèmes et des conséquences préoccupantes: l'extension possible de ces dispositions aux nouveau-nés et enfants handicapés, ou aux patients atteints de maladies psychiques, en est un exemple. En outre, dans les dernières années, des milliers de cas d'euthanasie active ont été rapportés, pratiqués au mépris de la demande expresse des patients. Certains politiciens hollandais ont même demandé que le patient s'exprime également clairement au sujet d'un refus d'euthanasie active: les plus grandes craintes sont permises au sujet de l'évolution en Hollande, où la "mort sur demande" (cf. travail de doctorat de la juriste Birgit Reuter [15]) est pratiquée par des médecins hollandais non seulement chez des malades qui n'étaient pas en mesure d'exprimer leur volonté, mais aussi sur des personnes capables de discernement à qui l'on n'avait pas demandé quoi que ce soit. Où cela va-t-il nous conduire? Ne devonsnous pas tirer des leçons des expériences faites en Hollande?

Du point de vue de l'éthique médicale judaïque, il est clair que des principes fondamentaux, tels que l'intangibilité et la sainteté de la vie humaine, de même que l'interdiction biblique de tuer et de se suicider, ne peuvent être abandonnés en aucun cas. L'histoire nous montre que la survie d'une civilisation dépend aussi de la force de ces valeurs morales. Notre société moderne, avec tous les progrès que la science, la médecine et la technologie, ont apportés, semble se diriger dans la voie dangereuse qui consiste à négliger les conceptions éthiques fondamentales. Il ne reste qu'à espérer que les arguments tels que ceux qui nous viennent de l'éthique médicale judaïque ne tombent pas dans l'oubli! 


\section{Références}

1 ben Maimom (Maimonides) M. Mischneh Tora, Rotseach, 2:2.

2 Schostak Z. Is there patient autonomy in halacha?

ASSIA, A journal of jewish medical ethics 1995;2,2:22-7.

3 Jakobovits I. Medical experimentation on humans in jewish law. In: Rosner F, Bleich JD (eds.). Jewish bioethics. New York: Hewbre Publishing; 1979. p. 377-83.

4 Leibowitz J, Shashar M. Gespräche über Gott und die Welt. Frankfurt am Main/Leipzig: Insel; 1994

5 Bleich JD. Judaism and healing. New York: Ktav; 1981.

6 ben Samuel J. Sefer Hachassidim, Nr. 273.

7 Isserles M. Kommentar zum Schulchan Aruch, Joreh Deah, 339:1.

8 Jakobovits I. Jewish medical ethics. New York: Bloch Publishing; 1975.
9 Waldenberg EJ. Ziz Elieser, V, 28:5, 29; IX, 47:5; X, 25:6.

10 Waldenberg EJ. Ziz Elieser, XIII, 87.

11 Waldenberg EJ. Ziz Elieser, XIV, 80.

12 Auerbach SS. In: Halacha Urefuah 2 (1981):131.

13 Feinstein M. Iggerot Mosche, Joreh Deah, 2, Nr. 174.

14 Abraham AS. Euthanasia. ASSIA, A journal of jewish medical ethics 1989;1,2:36-9.

15 Reuter B. Die gesetzliche Regelung der aktiven ärztlichen Sterbehilfe des Königreichs der Niederlande - ein Modell für die Bundesrepublik Deutschland? Frankfurt am Main: Peter Lang; 2001.

Cf. le livre d'Yves Nordmann: «Zwischen Leben und Tod - Aspekte der Jüdischen Medizinethik», Peter Lang Verlag, 1999/2000, avec nombreuses références. 